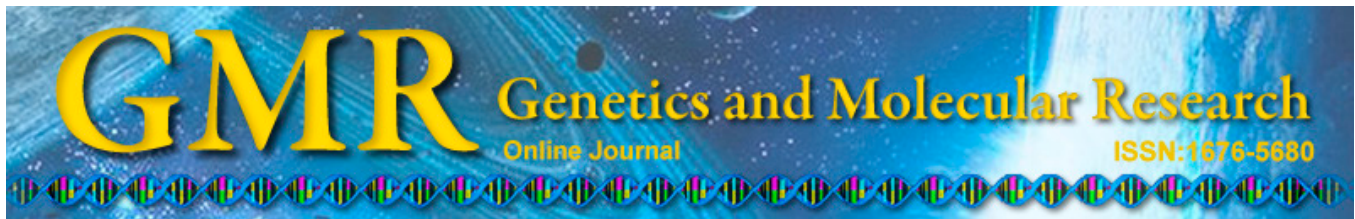

\title{
Mapping quantitative trait loci for nitrogen uptake and utilization efficiency in rice (Oryza sativa L.) at different nitrogen fertilizer levels
}

G.J. Dai ${ }^{1}$, S.H. Cheng ${ }^{2}$, Z.T. Hua ${ }^{3,4}$, M.L. Zhang ${ }^{1}$, H.B. Jiang ${ }^{1}$, Y. Feng', X.H. Shen ${ }^{2}$, Y.A. Su ${ }^{1}$, N. He ${ }^{1}$, Z.B. Ma ${ }^{1}$, X.Q. Ma ${ }^{1}$, S.G. Hou ${ }^{1}$ and Y.R. Wang ${ }^{1}$

${ }^{1}$ Rice Research Institute, Liaoning Academy of Agricultural Sciences, Shenyang, China

${ }^{2}$ China National Rice Research Institute, Hangzhou, China

${ }^{3}$ Tianjin University of Science \& Technology, Tianjin, China

${ }^{4}$ China National Japonica Rice Research and Development Center,

Tianjin, China

Corresponding author: Y.R. Wang

E-mail: wangyanrong518@163.com

Genet. Mol. Res. 14 (3): 10404-10414 (2015)

Received January 31, 2015

Accepted July 14, 2015

Published September 8, 2015

DOI http://dx.doi.org/10.4238/2015.September.8.1

\begin{abstract}
Genetic improvement is the fundamental basis for improving nitrogen-use efficiency. A better understanding of genetic factors controlling nitrogen uptake and utilization is required for crop genetic improvement. In this study, we identified the quantitative trait loci (QTLs) associated with traits of nitrogen uptake and utilization by using the single-sequence repeat marker method and a recombinant inbred line (RIL) population derived from a super hybrid Xieyou9308. All the traits investigated were inherited quantitatively by continuous variation and showed normal distribution in phenotype with transgressive segregation in the RIL population. Most of the traits were significantly correlated with each other except for nitrogen absorption ability
\end{abstract}


(NAA) with nitrogen harvest index (NHI) and NHI with agricultural nitrogen-absorption efficiency (ANAE). At logarithmic odds value of 2.3, total 13 candidate QTLs, including 4 for NAA, 2 for NHI, 2 for physiological nitrogen-use efficiency, 1 for agricultural nitrogen-use efficiency (ANUE), and 4 for ANAE, were detected and mapped on chromosomes 2, 3, 4, 5, 8, 9, 10, and 12. Significant pleiotropic effect or neighboring expression of QTLs was observed among traits. At position $64.8 \mathrm{cM}$ on chromosome 4 near the marker RM5757, there was a QTL cluster of NAA, ANUE, and ANAE, and at chromosome 5 near the marker RM5968, there was a QTL cluster of NAA and ANUE. The QTL clusters might provide partial explanation and genetic mechanism for the observed correlations between nitrogen uptake and utilization efficiency traits and might form a basis for future breeding programs.

Key words: Nitrogen uptake and utilization efficiency; QTL; Rice (Oryza sativa L.); Recombinant inbred line

\section{INTRODUCTION}

Given the limited arable land area, crop productivity should be improved continuously to meet food demand of the expanding human population. Nitrogen fertilizer is known to play an important role in the improvement of crop productivity (Xi, 2003). However, its usage in enhancing crop yield has been limited within a specific range. Once the nitrogen fertilizer amount applied exceeds a critical value, there is a decrease in crop yield, resulting in decreased fertilizer-use efficiency (Jiang et al., 2005). Moreover, excessive application of nitrogen fertilizer causes not only resource wasting, increased production costs, and pesticide application and poorer quality, but also a series of environmental problems such as the greenhouse effect, soil compaction, and groundwater pollution (Harrison et al., 1995; Edmunds and Gaye, 1997; Smith et al., 1997; Chen et al., 2001; Ju and Zhang, 2003; Zhu et al., 2007). Therefore, enhancing the nitrogen-use efficiency of crop varieties is an effective approach to address the conflicts among human population, resources, and environment by ensuring high crop productivity.

Rice (Oryza sativa L.) is a major food crop, fulfilling approximately $60 \%$ dietary requirement of the world population. In China, the nitrogen fertilizer application rate has reached an averaged value of $180 \mathrm{~kg} / \mathrm{hm}^{2}$, and even over $300 \mathrm{~kg} / \mathrm{hm}^{2}$ in some regions (Wang et al., 2006). Nevertheless, the nitrogen-use efficiency in rice is only $28-35 \%$, which is 15 $20 \%$ lower than the global average ( $\mathrm{Li}$ and Tang, 2006). Hence, there is a need to increase the nitrogen-use efficiency in China. In the soil-crop system, crops play a decisive role in nutrient uptake and utilization. Hence, genetic improvement of crops might be the most fundamental and effective way to improve nitrogen-use efficiency. Although significant genotypic differences in nitrogen uptake and utilization process have been widely reported (Piao et al., 2003; Ju et al., 2006; Lu et al., 2006; Zhao et al., 2012), no substantial progress has been achieved in the improvement of nitrogen-use efficiency of rice via genetic selection in breeding practice (Piao et al., 2004; Huang et al., 2006). This is probably because of the overall complex phenotype and its evaluation and lack of available genetic methods. 
With the development of molecular techniques and successful establishment of highdensity molecular genetic linkage map, quantitative trait locus (QTL) analysis has become an important method for genetic research of complex quantitative traits and has been widely used for most agronomic traits in rice (Nakagawa et al., 2005; Yoshinobu et al., 2007; Fujita et al., 2012; Liu et al., 2006; 2013). However, there are few reports on its application for investigating nitrogen uptake and utilization characteristics (Senthilvel, 1999; Senthilvel et al., 2001; Fang et al., 2001; Shan et al., 2005). In this study, the QTLs associated with nitrogen uptake and utilization characteristics in rice were mapped using a recombinant inbred line (RIL) population derived from the combination of XieqingzaoB/Zhonghui9308.

\section{MATERIAL AND METHODS}

\section{Plant materials and field experiments}

An RIL population consisting of 281 lines was constructed by crossing XieqingzaoB (XieB) and Zhonghui9308 (9308) and by using the single-seed descent method. The 2 parents show remarkable differences in nitrogen uptake and utilization characteristics. The F1 between XieB and 9308, named Xieyou9308, is an elite super hybrid rice combination released by the China National Rice Research Institute.

Phenotyping experiment was conducted at the breeding base in Sanya City of Hainan Island (China). The 2 parents (50 plants each) and 275 RILs randomly selected were grown from November 2008 to April 2009. The germinated seeds were sown on a seedling bed on November 20, and the seedlings were then transplanted to a paddy field on December 25, with one plant per hill spacing at $30 \times 13 \mathrm{~cm}$.

The experiment was performed in a split-plot design with 3 replications, with plot size of $2.4 \mathrm{~m}^{2}$. Three nitrogen levels were applied, i.e., $321 \mathrm{~kg} / \mathrm{hm}^{2}(\mathrm{H}), 208 \mathrm{~kg} / \mathrm{hm}^{2}(\mathrm{M})$, and $0 \mathrm{~kg} / \mathrm{hm}^{2}(\mathrm{~L})$. Other managements of the field experiment were in accordance with the local standard practices.

At physiological maturity stage, 3 plants per plot were sampled to measure grain weight (GW), straw weight (SW), N\% in stems and leaves (SN\%), and N\% in grain (GN\%). N\% was determined using the Kjeldahl method (Tang et al., 2004) in a reaction system containing $50 \mathrm{~mL} \mathrm{H} \mathrm{H}_{2} \mathrm{O}, 60 \mathrm{~mL} 2 \% \mathrm{HBO}_{3}$, and $100 \mathrm{~mL} 30 \% \mathrm{NaOH}$, with the digestion, distillation, and reaction times of $5 \mathrm{~s}, 1.5 \mathrm{~h}, 240 \mathrm{~s}$, respectively. The parameters nitrogen accumulation amount (NAA), nitrogen harvest index (NHI), physiological nitrogen-use efficiency (PNUE), agricultural nitrogen-use efficiency (ANUE), and agricultural nitrogen-absorption efficiency (ANAE) were calculated using Equations 1-5, where the $\mathrm{GW}_{0}$ and $\mathrm{N}_{0}$ represent the $\mathrm{GW}$ and $\mathrm{N}$ at L nitrogen level, respectively.

$\begin{array}{ll}N A A=I G W \times G N \% / / S W \times S N \% ! & (\text { Equation 1) } \\ N H l=G W \times G N \% / N A A & (\text { Equation 2) } \\ P N U E=G W / N A A & (\text { Equation 3) } \\ A N U E=I G W-G W, / N-N_{0}, & (\text { Equation 4) } \\ A N A E=+N A A-N A A_{0}, / N-N_{0} \cdot \times 100 & \text { (Equation 5) }\end{array}$




\section{DNA extraction and single-sequence repeat (SSR) marker analysis}

The seedlings of the 281 RILs and their parents were harvested at 3.5-leaf age for isolating genomic DNA by using the CTAB method (Murray and Thompson, 1980); this procedure was performed in Shenyang City. A total of 1000 SSR markers (Temnykh et al., 2000; Mccouch et al., 2002) evenly spanning 12 chromosomes were used for parental polymorphism analysis. In all, 196 markers showing abundant polymorphism were selected to genotype the RILs. Polymerase chain reaction (PCR) system included $25 \mathrm{ng}$ template DNA, 5 pmol of each primer, PCR buffer $\left(1.0 \mathrm{mM} \mathrm{MgCl}_{2}, 0.1 \mathrm{mM}\right.$ dNTPs, and $0.05 \mathrm{U}$ Taq polymerase) in total volume of $20 \mu \mathrm{L}$. PCR was performed on 96-well plates using a PTC200 thermocycler (MJ Research, Watertown, MA, USA). The reaction components were first predenatured at $94^{\circ} \mathrm{C}$ for $5 \mathrm{~min}$, followed by 35 cycles of $94^{\circ} \mathrm{C}$ for $1 \mathrm{~min}, 55^{\circ} \mathrm{C}$ for 1 min, and $72^{\circ} \mathrm{C}$ for $2 \mathrm{~min}$, with a final elongation at $72^{\circ} \mathrm{C}$ for $10 \mathrm{~min}$. The PCR products were separated on $6 \%$ non-denaturing polyacrylamide gel in $1 \mathrm{X}$ TBE at $150 \mathrm{~V}$ for $1 \mathrm{~h}$ by using the DYCZ-30b gel rig (Liuyi, Beijing, China). The amplified products were visualized using silver-staining method.

\section{Statistical analysis and QTL mapping}

The genetic linkage map was constructed using MAPMAKER/EXP 3.0 (Lander et al., 1987), with the heterozygous genotype as missing. The markers were classified into linkage groups with logarithm of odds (LOD) $=3.0$ and $\mathrm{LOD}=4.0$, and the order of markers within a linkage group was determined with $\mathrm{LOD}=2.0$. The physical distances of the markers were transformed into CentiMorgan (cM) using the Kosambi mapping function, and the chromosome number in linkage groups was determined according to the published map (Temnykh et al., 2000; McCouch et al., 2002). The genetic linkage map was drawn using the MapDraw V2.1 software.

QTL analysis was performed using Windows QTL Cartographer 2.5 (Wang et al., 2006) according to the method of composite interval mapping. An LOD score of 2.3 was set as a threshold for declaring the presence of QTL. The walking speed chosen for all QTL analysis was $1 \mathrm{cM}$. Forward \& Backward Method in Model 6: Standard Model was used for the background control, with $\mathrm{P}=0.05$ as the threshold. The multiple composite interval mapping method was used for the detection of epistatic QTLs.

\section{RESULTS}

\section{Phenotypic performance}

Phenotypic values of parents and distribution in RIL population are shown in Table 1, and the frequency distribution of the involved traits in the RIL population is shown in Figure 1. The 2 parents were significantly different $(\mathrm{P}<0.05$ or $\mathrm{P}<0.01)$ from each other in all the traits at $\mathrm{H}$ nitrogen level, in NHI and PNUE at M nitrogen level, but they did not differ significantly in most traits except for PNUE at L nitrogen condition. For most of the traits, the male parent 9308 was higher than the female parent XieB. For the RIL population, all the traits were inherited quantitatively, and transgressive segregation of traits was noted. 
Table 1. Phenotypic values of parents and distribution in recombinant inbred line (RIL) population.

\begin{tabular}{|c|c|c|c|c|c|c|c|c|c|}
\hline \multirow[t]{2}{*}{ Trait } & \multirow[t]{2}{*}{ Nitrogen fertilizer level } & \multicolumn{3}{|c|}{ Parents } & \multicolumn{5}{|c|}{ RIL population } \\
\hline & & $\mathrm{XieB}$ & 9308 & $\mathrm{~F}$ & Min & $\operatorname{Max}$ & Mean & Skew & Kurtosis \\
\hline \multirow[t]{3}{*}{ NAA } & $\mathrm{H}$ & 0.4673 & 0.5062 & $58.4 * *$ & 0.230 & 0.841 & 0.451 & 0.574 & 0.523 \\
\hline & M & 0.3289 & 0.3550 & 8.15 & 0.164 & 0.770 & 0.358 & 1.139 & 2.595 \\
\hline & $\mathrm{L}$ & 0.2305 & 0.2188 & 1.76 & 0.104 & 0.423 & 0.184 & 1.791 & 7.613 \\
\hline \multirow[t]{3}{*}{ NHI } & $\mathrm{H}$ & 0.6900 & 0.7479 & $19.11^{*}$ & 0.363 & 0.818 & 0.646 & -0.259 & 0.040 \\
\hline & M & 0.7157 & 0.8245 & $685.0 * *$ & 0.479 & 0.931 & 0.670 & 0.074 & 0.557 \\
\hline & $\mathrm{L}$ & 0.6537 & 0.6246 & 6.52 & 0.466 & 0.891 & 0.697 & -0.610 & 1.169 \\
\hline \multirow[t]{3}{*}{ PNUE } & $\mathrm{H}$ & 50.94 & 57.60 & $43.9 *$ & 29.55 & 78.24 & 53.42 & 0.135 & 0.276 \\
\hline & M & 61.01 & 69.37 & $561.0^{* *}$ & 37.67 & 88.93 & 59.89 & 0.230 & 0.592 \\
\hline & $\mathrm{L}$ & 55.91 & 69.92 & $96.3 * *$ & 40.32 & 91.22 & 67.33 & 0.008 & 0.072 \\
\hline \multirow[t]{2}{*}{ ANUE } & $\mathrm{H}$ & 7.73 & 9.33 & $54.7 *$ & 0.37 & 26.54 & 9.29 & 0.614 & 0.601 \\
\hline & M & 7.08 & 8.50 & 4.01 & -7.68 & 37.04 & 10.80 & 0.519 & 2.235 \\
\hline \multirow[t]{2}{*}{ ANAE } & $\mathrm{H}$ & 17.84 & 21.51 & $80.4^{* *}$ & 3.64 & 50.03 & 21.50 & 0.492 & 0.249 \\
\hline & M & 10.02 & 13.95 & 12.66 & -8.9 & 58.78 & 21.00 & 0.497 & 1.412 \\
\hline
\end{tabular}

*,**Indicate significant level at $\mathrm{P}<0.05$ and $\mathrm{P}<0.01$, respectively. The same as below.
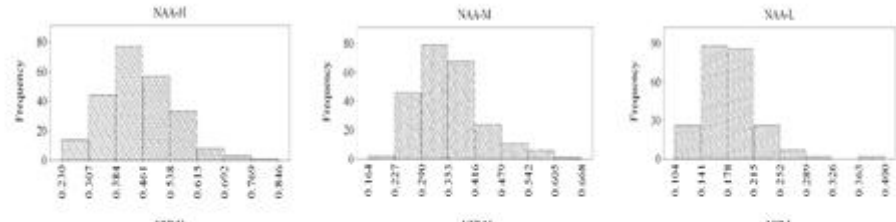

NHE

NEL
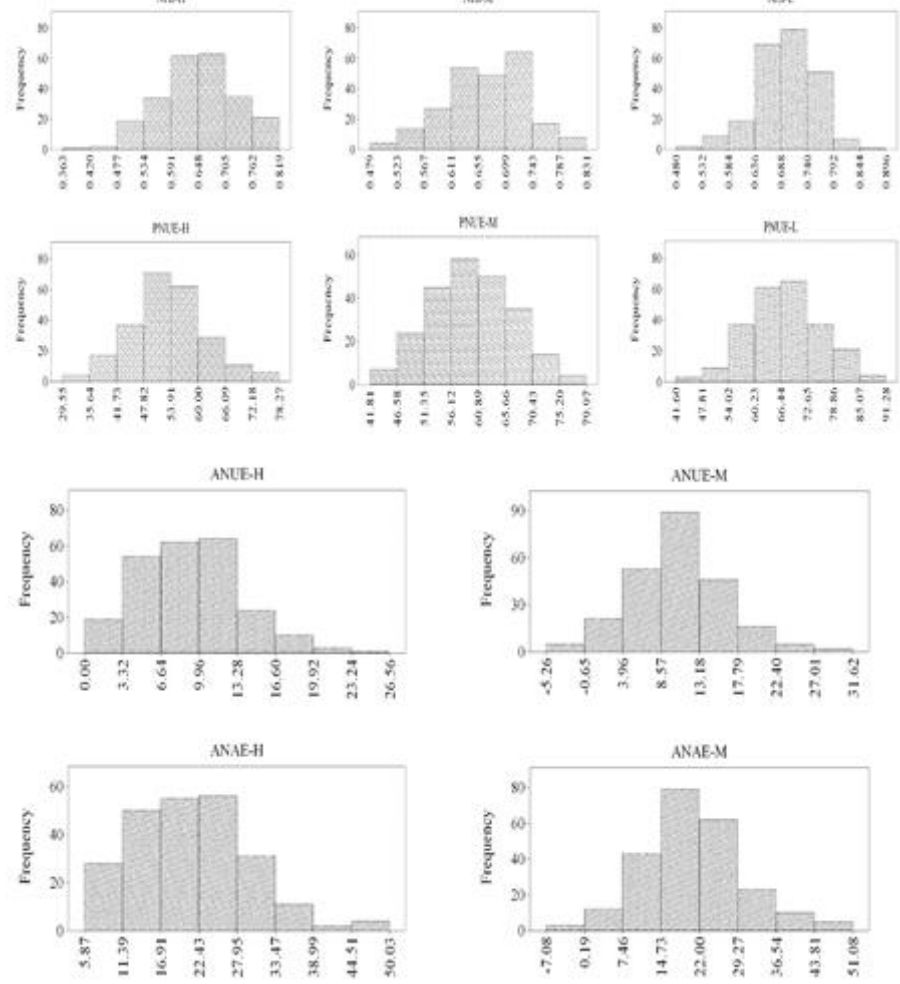

Figure 1. Frequency distribution of the involved traits in the recombinant inbred line population. 
The averaged values of the RIL population were within the range of parental value in most of the traits except for NAA, NHI, and ANAE. However, for NAA at H and L nitrogen levels and for NHI at $\mathrm{H}$ and $\mathrm{M}$ nitrogen levels, the averaged value of the RIL population was smaller than the lower parental value. On the other hand, for NAA at M nitrogen level, for NHI at L nitrogen level, and for ANAE at $\mathrm{H}$ and $\mathrm{M}$ nitrogen levels, the averaged value was above the higher parental value. Most traits except for NAA at M and L nitrogen levels showed a normal distribution with skew and kurtosis less than or close to 1 or -1 .

\section{Correlation analysis}

The correlation coefficients between the traits in the RIL population were calculated and are listed in Table 2. Most of the traits were significantly correlated with each other $(\mathrm{P}<$ 0.01 ) except for NAA with NHI and NHI with ANAE at all nitrogen levels. Most traits were positively correlated with each other except for NAA with PNUE and PNUE with ANAE. This suggests that trait selection can be realized via other closely correlated traits.

\begin{tabular}{|c|c|c|c|c|}
\hline Trait & NAA & $\mathrm{NHI}$ & PNUE & ANUE \\
\hline \multicolumn{5}{|l|}{$\mathrm{H}$} \\
\hline NHI & -0.103 & & & \\
\hline PNUE & $-0.278^{* *}$ & $0.742 * *$ & & \\
\hline ANUE & $0.713^{* *}$ & $0.330 * *$ & $0.292 * *$ & \\
\hline ANAE & $0.921 * *$ & -0.115 & $-0.294 * *$ & $0.784 * *$ \\
\hline \multicolumn{5}{|l|}{ M } \\
\hline NHI & 0.107 & & & \\
\hline PNUE & $-0.264^{* *}$ & $0.613 * *$ & & \\
\hline ANUE & $0.716^{* *}$ & $0.365^{* *}$ & $0.200^{* *}$ & \\
\hline ANAE & $0.878 * *$ & 0.080 & $-0.254 * *$ & $0.842 * *$ \\
\hline \multicolumn{5}{|l|}{ L } \\
\hline NHI & -0.047 & & & \\
\hline PNUE & $-0.189 * *$ & $0.719 * *$ & & \\
\hline
\end{tabular}

\section{QTL detection}

Putative QTLs controlling the traits detected in the RIL population are shown in Table 3 , and location of QTLs for the involved traits at different $\mathrm{N}$ levels on the linkage map is shown in Figure 2. A total of 13 putative QTLs controlling the 5 traits were detected on chromosomes $2,3,4,5,8,9,10$, and 12 , with additive effects.

Four QTLs for NAA, including qNAA4, qNAA5, qNAA10, and qNAA12, were detected near the markers RM5757, RM5968, RM6142, and RM5364 on chromosomes 4, 5, 10, and 12, with LOD scores of 3.2, 2.9, 4.1, and 2.4, respectively. Among them, qNAA12 was detected at $\mathrm{H}$ nitrogen level accounting for $4.3 \%$ of phenotypic variation; the other 3 were detected at the $\mathrm{M}$ nitrogen level, accounting for $19.3 \%$ of phenotypic variation together. qNAA4, qNAA5, and qNAA12 had positive alleles from 9308, and qNAA10 had positive alleles from XieB.

Two QTLs for NHI were detected on chromosomes 2 and 12 near the makers RM5812 and RM7003 at $\mathrm{H}$ and $\mathrm{M}$ nitrogen levels and were named qNHI2 and qNHI12, respectively. qNHI12 and qNHI2 accounted for 5.3 and $4.7 \%$ of phenotypic variation, respectively, and both had positive alleles from 9308. 
Table 3. Putative QTLs controlling the traits detected in the recombinant inbred line (RIL) population.

\begin{tabular}{|c|c|c|c|c|c|c|c|c|}
\hline Trait & $\mathrm{N}$ & QTL & Chromosome & Marker & Position & LOD & ADD & $\mathrm{R}^{2}(\%)$ \\
\hline \multirow[t]{4}{*}{ NAA } & $\mathrm{H}$ & qNAA12 & 12 & RM5364 & 47.4 & 2.4 & 0.02 & 4.3 \\
\hline & M & qNAA4 & 4 & RM5757 & 64.8 & 3.2 & 0.02 & 5.7 \\
\hline & & qNAA5 & 5 & RM5968 & 176.8 & 2.9 & 0.02 & 5.0 \\
\hline & & qNAA10 & 10 & RM6142 & 58.5 & 4.1 & -0.02 & 8.6 \\
\hline \multirow[t]{2}{*}{ NHI } & $\mathrm{H}$ & qNHI12 & 12 & RM7003 & 6.0 & 2.7 & 0.02 & 5.3 \\
\hline & M & qNHI2 & 2 & RM5812 & 91.9 & 2.4 & 0.01 & 4.7 \\
\hline \multirow[t]{2}{*}{ PNUE } & $\mathrm{L}$ & qPNUE3 & 3 & RM5761 & 16.0 & 2.5 & 2.22 & 6.5 \\
\hline & & qPNUE4 & 4 & RM1205 & 38.5 & 2.5 & 1.81 & 4.5 \\
\hline ANUE & M & qANUE4 & 4 & RM5757 & 64.8 & 2.3 & 1.19 & 4.4 \\
\hline \multirow[t]{4}{*}{ ANAE } & M & qANAE4 & 4 & RM5757 & 64.8 & 3.7 & 2.53 & 6.7 \\
\hline & & qANAE5 & 5 & RM5968 & 174.8 & 2.3 & 1.99 & 4.2 \\
\hline & & qANAE8 & 8 & RM5485 & 116.0 & 2.4 & -2.1 & 4.8 \\
\hline & & qANAE9 & 9 & RM6491 & 78.1 & 2.3 & 2.06 & 4.6 \\
\hline
\end{tabular}

Marker $=$ the nearest marker to the QTL; ADD $=$ additive effects, a negative sign means that the positive alleles come from the parent XieB, while a positive sign means that positive alleles come from the parent $9308 ; \mathrm{R}^{2}(\%)=$ phenotypic variation explained.
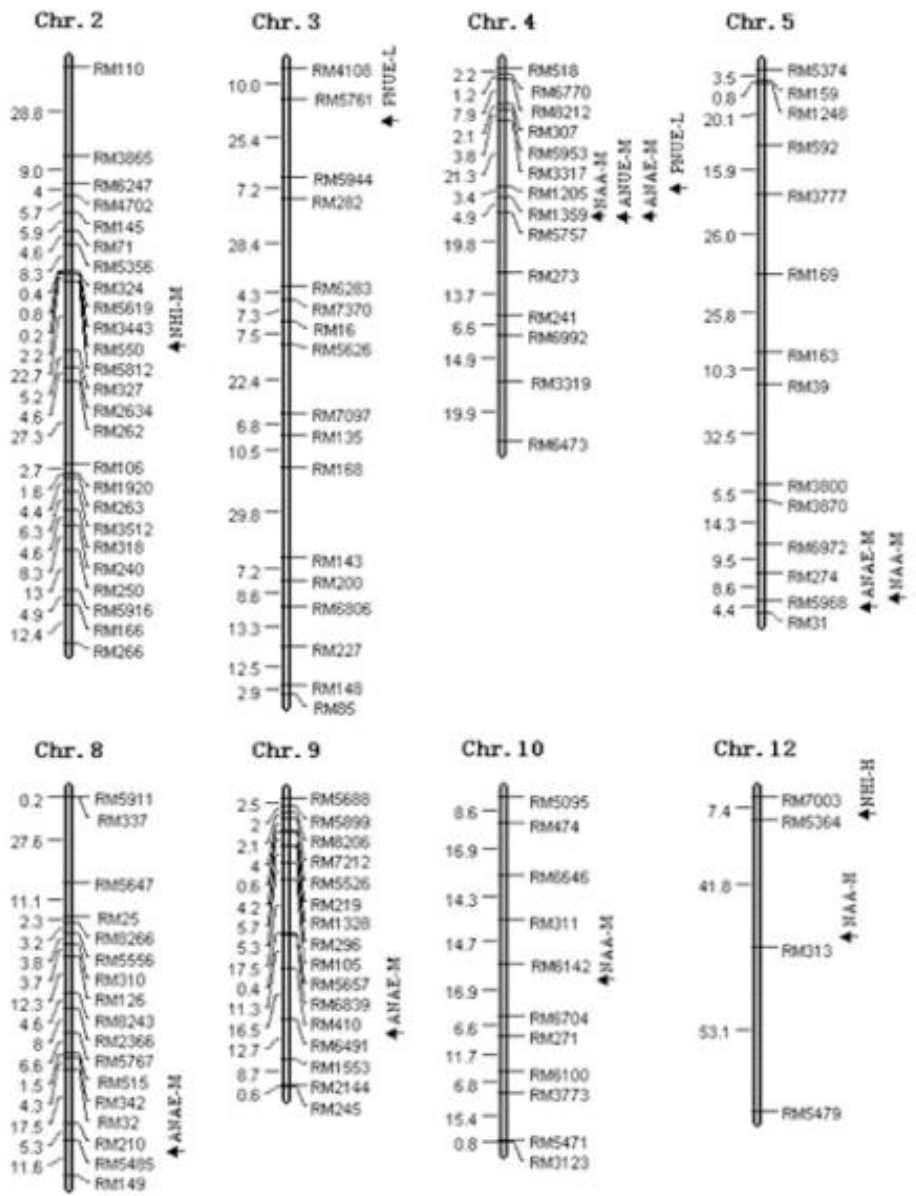

Figure 2. Location of QTLs for the involved traits at different $\mathrm{N}$ levels on the linkage map. 
Two QTLs for PNUE were detected only at L nitrogen level on chromosomes 3 and 4 near the markers RM5761 and RM1205 and were named qPNUE3 and qPNUE4, respectively. qPNUE3 and qPNUE4 both had positive alleles from 9308 and accounted for $11 \%$ of phenotypic variation together.

Only one QTL was detected at M nitrogen level for ANUE on chromosome 4 near the marker RM5757 and was named qANUE4. qANUE4 had positive alleles from 9308 and accounted for $4.4 \%$ of phenotypic variation.

Four QTLs for ANAE were detected only at M nitrogen level on chromosomes 4, 5, 8, and 9 near the markers RM5757, RM5968, RM5485, and RM6491 and were named qANAE4, qANAE5, qANAE8, and qANAE9, respectively. These accounted for $20.3 \%$ of phenotypic variation together. Only qANAE8 had positive alleles from XieB, the others had positive alleles from 9308 .

The QTLs detected varied among different nitrogen levels. For NAA and NHI, QTLs were detected only at $\mathrm{H}$ and $\mathrm{M}$ nitrogen levels; for PNUE, only at $\mathrm{L}$ nitrogen condition; and for ANUE and ANAE, only at M nitrogen level. Among the traits, there were significant pleiotropic effects of QTL, that is, one QTL controlled more than one trait. At the position $64.8 \mathrm{cM}$ on chromosome 4 near the marker RM5757, QTLs including qNAA4, qANUE4, and qANAE4 overlapped with each other. In addition, NAA and ANUE had QTLs close to each other, that is, qNAA5 and qANUE5. The traits with overlapped or adjacent QTLs might be more closely related to each other.

\section{DISCUSSION}

A better understanding of genetics is the basis for the genetic improvement of traits. Plant nitrogen uptake and use efficiencies have been proved to be quantitative traits that are controlled by multiple genes (Olivier et al., 2003). Nitrogen-use efficiency has been thought to follow the additive effect model under normal nitrogen levels and the non-additive effect model under low nitrogen levels (Piao et al., 2005; Hao et al., 2006). According to the results of $\mathrm{Li}$ et al. (2006), nitrogen uptake ability and nitrogen-use efficiency of rice were controlled by 2 major loci and multiple minor ones. Fang et al. (2001) detected both additive and dominant effects on nitrogen-use efficiency. Our results confirmed the quantitative characteristics of all the nitrogen uptake and utilization traits from continuous variation in the RIL population, but just the additive effect was observed.

QTL is a powerful tool for genetic analysis of complex quantitative traits. Fang et al. (2001) detected 1 QTL for NUE (PNUE of biomass) on chromosome 12 at the interval of RG241-RG264 at seedling stage, accounting for 18\% of phenotypic variation. By using an F2 population, Senthilvel et al. (2001) detected 4 QTLs for TNUP (NAA) on chromosomes 3, 3, 4, and 8 near the markers RM282, RM293, RM317, and RM38, accounting for $15.7 \%$ of phenotypic variation together; and 5 QTLs for NUE (PNUE) on chromosomes 1, 3, 6, 7, and 11 near the markers RM246, RM293, RM225, RM2, and RM202, accounting for 15\% of phenotypic variation together. By using an RIL population, Shan et al. (2005) detected 2 QTLs for WPNA (NAA) on chromosomes 4 and 6 at the interval of G102-RM255 and RM225-C226, accounting for $23.2 \%$ of phenotypic variation together; and 1 QTL for NUEp (PNUE of biomass) on chromosome 6 at the interval of Waxy-C1496, accounting for $12.9 \%$ of phenotypic variation; this QTL was not on the same chromosome as that detected by Fang et al. (2001). In 
this study, the QTLs controlling nitrogen uptake and utilization characteristics were analyzed at different nitrogen levels by using an RIL population from XieB/9308. For NAA, 1 QTL was detected at $\mathrm{H}$ nitrogen level on chromosome 12 near the marker RM5364, accounting for $4.3 \%$ of phenotypic variation; 3 QTLs were detected at M nitrogen level on chromosomes 4, 5, and 10 near the markers RM5757, RM5968, and RM6142, accounting for $19.3 \%$ of phenotypic variation together. Although 1 QTL was detected on chromosome 4, which was similar to the result of Senthilvel et al. (2001) and Shan et al. (2005), the QTLs detected by different research groups were not on the same position. For NHI, 1 QTL was detected at H and M nitrogen levels, respectively, on chromosomes 12 and 2 near the markers RM7003 and RM5812, accounting for 5.3 and $4.7 \%$ of phenotypic variation, respectively. There were 2 QTLs for PNUE detected only at L nitrogen level on chromosomes 3 and 4 near the markers RM5761 and RM1205, accounting for $11 \%$ of phenotypic variation together. Only 1 QTL was detected for ANUE at M nitrogen level on chromosome 4 near the marker RM5757, accounting for $4.4 \%$ of phenotypic variation. Four QTLs for ANAE were detected only at M nitrogen level on chromosomes 4, 5, 8, and 9 near the markers RM5757, RM5968, RM5485, and RM6491, accounting for 20.3\% of phenotypic variation together. With regard to a specific trait, QTL mapping results varied among different research groups, mainly depending on the materials and methods adopted. Therefore, the veracity and practicability of a detected QTL should be verified continuously and repeatedly. Further, the expression of quantitative characteristics is usually affected remarkably by environmental factors. In this study, various QTLs were detected under different nitrogen fertilizer conditions; therefore, further QTL-based marker-assisted selection should be conducted under the corresponding conditions.

Nitrogen uptake and utilization are the 2 continuous processes deciding nitrogen-use efficiency and are closely correlated with each other. According to the correlation analysis results in this study, NAA and ANAE were significantly correlated with PNUE and ANUE, but not with NHI. This suggests that nitrogen uptake has little relation with the distribution of nitrogen in grains. Among the QTLs associated with NAA, ANAE, and ANUE detected in this study, 1 was overlapped, simultaneously controlling these 3 traits, and 1 for NAA expressed near a QTL for ANAE. This indicates that the traits with overlapped or neighboring QTLs have more close correlation.

Nitrogen uptake and utilization are complex quantitative characteristics that are difficult to study and evaluate. The QTLs detected in this study can be used to reveal the genetic mechanism of nitrogen uptake and utilization and provide evidence for further markerassisted selection in nitrogen-use efficiency improvement, especially those with higher LOD values. Despite the results were based on data collected during 1 year in this study and have to be verified further and repeatedly, they have certain guiding significance, because the RIL population adopted was considerably typical from the elite super hybrid rice combination Xieyou9308, with the 2 parents differing significantly in nitrogen uptake and utilization characteristics. Further, the experiments were performed at different nitrogen levels, and hence, the results should be applied in a more targeted manner.

\section{ACKNOWLEDGMENTS}

Research supported by a grant from the Engineering Research Center of Liaoning Province of China (\#2010402016); the Liaoning Provincial Natural Science Foundation of China (\#201102102); and the National "863" Program of China (\#2010AA101303). 


\section{REFERENCES}

Chen GC, Gan L, Wang SL and Wan GJ (2001). Soil nitrogen and its environmental effects. Geol. Geochem. 1: 63-67.

Edmunds WM and Gaye CB (1997). Naturally high nitrate concentration in groundwater from the Sahel. J. Environm. Qual. 26: 1231-1239.

Fang P, Tao QN and Wu P (2001). QTLs underlying rice root to uptake $\mathrm{NH}_{4}-\mathrm{N}$ and $\mathrm{NO}_{3}-\mathrm{N}$ and rice $\mathrm{N}$ use efficiency at seedling stage. Plant Nutr. Fertilizer Sci. 2: 159-165.

Fujita D, Tagle AG, Ebron LA, Fukuta Y, et al. (2012). Characterization of near-isogenic lines carrying QTL for high spikelet number with the genetic background of an indica rice variety IR64 (Oryza sativa. L). Breed. Sci. 62: 18-26.

Hao X, Dong ZD, Li Y and Cui DQ (2006). Genetic model of nitrogen concentrations and nitrogen utilization efficiency of wheat in different water and nitrogen environment. J. Triticeae Crops 4: 103-106.

Harrison RM, Yamulki S and Goulding KWT (1995). Effects of fertilizer application on $\mathrm{NO}$ and $\mathrm{N}_{2} \mathrm{O}$ fluxes from agricultural fields. J. Geophys. Sci. 100: 25923-25931.

Huang NR, Zhong XH and Zheng HB (2006). Selection of rice genotypes with high nitrogen utilization efficiency and its evaluation indices. Chin. Agric. Sci. Bull. 6: 29-34.

Jiang J, Huang Y and Yang ZF (2005). A statisical model of nitrogen use efficiency for rice and wheat crops. J. Nanjing Agric. Univ. 1: 57-60.

Ju J, Yamamoto Y, Wang YL, Shan YH, et al. (2006). Genotypic difference in grain yield, and nitrogen absorption and utilization in recombinant inbred lines of rice under hydroponic culture. Soil Sci. Plant Nutr. 52: 321-330.

Ju XT and Zhang FS (2003). Nitrate accumulation and its implication to environment in north China. Ecol. Environm. 1: 24-28.

Lander ES, Green P, Abrahamson, Barlow A, et al. (1987). MAPMAKER: an interactive computer package for constructing primary genetic linkage maps of experimental and natural populations. Genomics 1: 174-181.

Li H and Tang QY (2006). Nitrogen-use efficiency of rice and its research progress in China. Crop Res. 5: 401-408.

Li YH, Wang ZF, Guan RZ, Wang JF, et al. (2006). Genetic analysis on nitrogen uptake and utilization efficiency of seedlings in rice. Chin. J. Rice Sci. 4: 379-383.

Liu GF, Yang J and Zhu J (2006). Mapping QTL for biomass yield and its components in rice (Oryza sativa L.). Acta Genet. Sin. 7: 607-616.

Liu QM, Jiang JH, Niu FA, He YJ, et al. (2013). QTL analysis for seven quality traits of RIL population in japonica rice based on three genetic statistial models. Rice Sci. 1: 31-38.

Lu YL, Lu WP, Wang JF, Liu P, et al. (2006). The differences of nitrogen uptake and utilization in waxy corn. The different genotypes in absorption and utilization of nitrogen. Plant Nutr. Fertilizer Sci. 3: 321-326.

McCouch SR, Teytelman L, Xu Y, Lobos KB, et al. (2002). Development and mapping of 2240 new SSR markers for rice (Oryza sativa L.). DNA Res. 9: 199-207.

Murray MG and Thompson WF (1980). Rapid isolation of high molecular weight plant DNA. Nucleic Acids Res. 8: 43214325.

Nakagawa H, Yamagishi J, Miyamoto N, Motoyama M, et al. (2005). Flowering response of rice to photoperiod and temperature - a QTL analysis using a phenological model. Theor. Appl. Genet. 110: 778-786.

Olivier L, Sylvain C, Patricia M, Joel T, et al. (2003). Quantitative trait loci analysis of nitrogen use efficiency in Arabidopsis. Plant Physiol. 131: 345-358.

Piao ZZ, Han LZ and Gao XZ (2003). Variations of nitrogen use efficiency by rice genotype. Chin. J. Rice Sci. 3: 233-238.

Piao ZZ, Han LZ, Gao XZ, Lu JA, et al. (2004). Selection effect of nitrogen use efficiency in rice. Acta Agronom. Sin. 7: 651-656.

Piao ZZ, Han LZ, Gao XZ, Zhang JM, et al. (2005). Analysis on combining ability of dry weight and nitrogen use efficiency in rice. Chin. J. Rice Sci. 6: 527-532.

Senthilvel S (1999). Identification of quantitative trait loci associated with traits of agronomic importance, nitrogen uptake, and nitrogen use efficiency in rice (Oryza sativa L.). M.Sc. thesis, Tamil Nadu Agricultural University, Coimbatore, 65-70.

Senthilvel S, Govindaraj P, Arumugachamy S, Latha R, et al. (2001). Mapping genetic loci associated with nitrogen use efficiency in rice. In: 4th International Crop Science Congress. Available at [http://www.corpscience.org.au].

Shan YH, Wang YL and Pan XB (2005). Mapping of QTLs for nitrogen use efficiency and related traits in rice (Oryza sativa L.). Agric. Sci. China 10: 721-727.

Smith KA, McTaggart IP and Tsurata $\mathrm{H}$ (1997). Emissions of $\mathrm{N}_{2} \mathrm{O}$ and $\mathrm{NO}$ associated with nitrogen fertilization in intensive agriculture, and the potential for mitigation. Soil Use Manag. 13: 296-304.

Tang ZC, Wei JM, Chen Y and Zhong ZX (2004). Morden Plant Physiology Test Guide. Science Press, Beijin. 
Temnykh S, Park WD, Ayres N, Cartinhour S, et al. (2000). Mapping and genome organization of microsatellite sequence in rice (Oryza sativa L.). Theor. Appl. Genet. 100: 697-712.

Wang L, Du S, Wang JQ, Gao XZ, et al. (2006). Consumption situation of nitrogen fertilizers in China and prospects for development. Chem. Fertilizer Ind. 34: 1-6.

Xi ZB (2003). Chemical fertilizer and agriculture. Lin B. Chemical fertilizer and no-pollution agriculture. China Agricultural Press, Beijing, 159-174.

Yoshinobu T, Yasunori N, Takeshi E and Keitaro S (2007). QTL detection for eating quality including glossiness, stickiness, taste and hardness of cooked rice. Breed. Sci. 57: 231-242.

Zhao SP, Zhao XQ and Shi WM (2012). Genotype variation in grain yield response to basal N fertilizer supply among different rice cultivars. Afr. J. Biotechnol. 24: 12298-12304.

Zhu HX, Wang ZY, Dong Y and Hu XF (2007). Effects of fertilizer on rice quality. Soil Fertilizer Sci. China 1: 9-12. 